\title{
FINITE ELEMENT SIMULATION OF CRACK PROPAGATION AND DELAMINATION IN LAYERED SHELLS DUE TO BLADE CUTTING
}

\author{
Federica Confalonieri, Aldo Ghisi, and Umberto Perego \\ Department of Civil and Environmental Engineering, Politecnico di Milano, \\ piazza Leonardo da Vinci 32, 20133 Milano, Italy \\ e-mail: \{federica.confalonieri, aldo.ghisi, umberto.perego\}@polimi.it
}

Keywords: Blade cutting, crack propagation, delamination, explicit dynamics, finite elements, directional cohesive elements.

\begin{abstract}
This work is focused on some computational issues concerning the simulation of blade cutting of thin walled structures. In comparison to plain crack propagation problems, the presence of the blade brings in additional complexity, mainly due to the interaction between the blade and the material in the crack process zone. The blade sharpness introduces in the problem an extremely small geometrical scale (orders of magnitude smaller than a typical element in-plane size) that is here resolved using the so called directional cohesive elements, recently proposed in [6. 12]]. A special feature of this type of problems is that the crack path is driven by the blade trajectory, which is prescribed and hence known in advance. Crack propagation is therefore modeled by adjusting the mesh in such a way that shell element edges are disposed along the expected main crack path and then by interposing directional cohesive elements between the sides of separating elements. The transition from a continuous mesh to a mesh containing a crack with a cohesive interface is well known to be critical for the solution accuracy. Nodal equilibrium is in general violated during the transition, with subsequent generation of spurious stress oscillations that, in view of the non-reversible nature of the problem, can lead to significant deviations in the stress response. This aspect is investigated in this contribution. The effect of the number of introduced directional cohesive elements per opening face is critically assessed and a simple correction, based on an automatic adaptation of the cohesive model, is proposed. Numerical tests taken from the literature are used to validate the proposed approach.
\end{abstract}




\section{INTRODUCTION}

For the cutting simulation of thin shells, standard cohesive elements are not well suited to correctly address the interaction of the cohesive zone with a sharp blade. When a cutting tool interacts with a shell structure made of a ductile material, the blade curvature radius (sometimes of the order of micrometers) turns out to be far smaller than the typical size of a computationally acceptable in-plane discretization of the process zone, so that the interaction cannot be properly resolved. The blade can interfere with the correct transmission of the cohesive forces, leading to severe underestimation of the dissipated energy. A possible remedy to this inconvenience has been proposed in [6, 12], with the introduction of the so called directional cohesive elements (DCEs). When a crack propagation criterion is met at a node, the node is duplicated and cohesive string elements are attached to the opening faces. The main feature of these string elements is that they are able to detect contact with the cutting blade. Upon contact, the string element interacts with the cutting blade and deforms, transmitting cohesive forces to the two crack flanks in the correct directions. The correct amount of cohesive energy is therefore dissipated through the string elongation (see Figure 1).

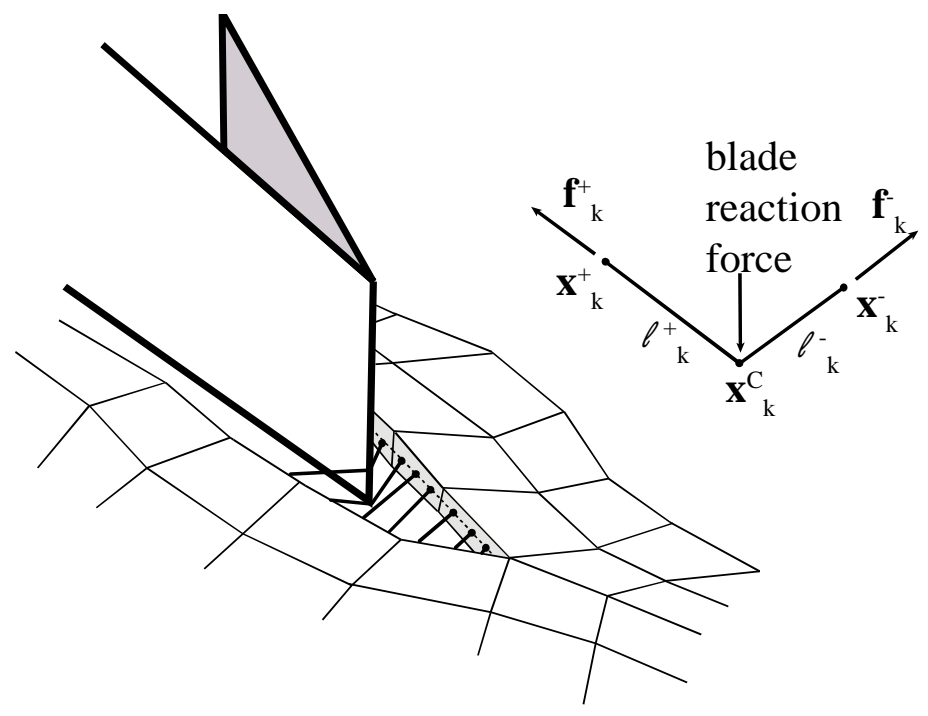

Figure 1: distribution of cohesive forces via the "directional" concept.

Despite the improved energy balance in the presence of a cutting blade, the issue of the accuracy of the cohesive zone discretization remains open. Another important issue arising during crack propagation is the oscillation in the stress field generated by the sudden release of a node at the crack tip. The node separation with the simultaneous introduction of the directional cohesive elements may generate a propagating shock, with severe stress oscillations. In the exact instant of the (directional or classical) cohesive element insertion, the nodal force balance is not automatically assured, because of the configuration change due to the appearing of new surfaces where cohesive forces have to be applied. These problems, which are present also with standard cohesive elements, are well known and have been discussed extensively in the literature on finite element cohesive models. Shellekens and De Borst [14] interpreted the oscillations as due to the combination of high tractions gradients and of the chosen integration scheme: in their examples, Gaussian integration (GI) for linear plane interface elements resulted in non-zero energy modes with coupling between degrees of freedom of individual node sets. 
They found that the Newton-Cotes integration (NCI) rule performed better even for quadratic cohesive elements and ascribed the responsibility to the integration point located at the face centroid. Alfano and Crisfield [1], however, showed that a higher order of the numerical integration is not sufficient to allow for a coarser mesh discretization neither guarantee the robustness of the solution algorithm which, conversely, could be negatively affected.

Recently, Gillormini and Diani [8] discussed the effect of the numerical integration rule in case of finite strain. While finite strain formulations for cohesive elements have been widely used (see e.g. [11,7]), these authors point out the influence of the choice of the reference configuration (original or current) where the numerical integration is carried out, and the consequence of neglecting the local basis mobility in the finite element formulation. The latter choice, in particular, leads to completely neglect a term in the traction-separation law: this is legitimate only in small deformations or whether tractions and displacements are collinear, such as in the present work. Their numerical peeling experiment shows no sensible differences between NCI and GI rules in nonlinear regimes, while a less precise result has been observed for NCI in the elastic part of the cohesive law.

The possibility to exploit numerical integration to improve the cohesive element computational efficiency has been investigated by Yang et al. [18], who introduced a cohesive element featuring a multiple subdomain integration: the numerical integration for each cohesive finite element is subdivided in zones whose dimensions are smaller than the cohesive zone size and inside which GI or NCI schemes are carried out. This approach makes it possible to increase the mesh size from a fraction (typically $\frac{1}{3} \div \frac{1}{5}$ ) of the cohesive zone to a size comparable and even slightly larger of the cohesive zone. Moreover, the modifications in a standard finite element implementation are quite limited, see [13]. In addition, it has been shown that it is possible to move the evolving crack front within a partially failed cohesive element if an improved stress integration scheme (GI or NCI) is implemented. Roughly speaking, the integration points are in this case moved ahead of the crack tip position, and the integrals for the cohesive model are then calculated on the uncracked domain restriction of the finite element traversed by the crack front. Even in this case, the outcome is the relaxation of the restraint on the minimum mesh size with respect to the cohesive zone.

To mitigate the oscillations due to unbalanced nodal forces upon the insertion of a new cohesive segment, Menouillard and Belytschko [10], for extended finite elements (XFEM), proposed to add artificial correction nodal forces. In their correction method, to reduce the jump due to the additionally injected enriched dofs, fictitious forces are added to the interested nodes to re-establish equilibrium. These forces are then scaled down when the crack tip reaches the next element edge in the propagation path.

It is well known that in finite element cohesive propagation, the cohesive energy is the critical parameter to be respected in the modeling of the cohesive properties, whereas the cohesive strength, the limit opening and the exact shape of the cohesive traction-separation law have a far smaller impact on accuracy. The adaptive definition of these latter parameters has also been proposed as a technique to improve accuracy in the presence of coarse finite element discretizations of the process zone [17, 16]. In view of these considerations, a correction similar to the one proposed in [9, 10] is considered in this work. The required correction force is calculated at the node in correspondence of the newly separating element crack tip, so as to compensate the missing force contribution coming from the adjacent element before separation. The additional contribution is however provided by a modification of the maximum traction and limit opening in the cohesive law, while preserving the cohesive energy.

The correction has been implemented in an explicit dynamics solid-shell finite element code 
together with the DCE technique. The effect on accuracy of the number of introduced string elements and of the proposed correction is investigated by application to test cases taken from the literature. In the next section 2 the basics of the DCEs, the rules for the numerical integration leading to the cohesive forces for multiple cables, and the adopted correction scheme are briefly summarized, while in section 3 we exemplify through simple cases the effects of our choices. Our conclusions are drawn in section 4. In all the paper, bold characters are reserved for vectors or matrices.

\section{FORMULATION}

Let us consider a continuum with the thickness smaller than the other dimensions. The body occupies a volume $\Omega_{0}$ in a reference configuration $\mathcal{B}_{0}$; its boundary is called $\partial \Omega_{0}$. The map of the deformation consequent to a change of configuration is $\chi(\mathbf{X}, t), t \in[0, T], \mathbf{X}$ being the original coordinates of a generic point in $\Omega_{0}$, and $T$ the time duration of of the process. After the change, the body occupies the volume $\Omega$ in the current deformed configuration $\mathcal{B}$. A cohesive crack through the thickness is described via the original interface $\Gamma_{0}$, which evolves into $\Gamma$ because of the configuration change. Across the surface $\Gamma$, displacement discontinuities are defined as $\delta=\mathrm{x}^{+}-\mathrm{x}^{-}$, being $\mathrm{x}^{+}$and $\mathrm{x}^{-}$the current coordinates of the material points belonging to the flank surface originally linked and then separating into $\Gamma^{+}$and $\Gamma^{-}$. A variational balance in dynamics, neglecting damping, accounting for the change in kinetic, internal energy and external work is written as:

$\delta \Pi_{\mathrm{kin}}^{e}+\delta \Pi_{\mathrm{int}}^{e}-\delta \Pi_{\mathrm{ext}}^{e}=\left[\int_{\Omega} \rho \ddot{\mathbf{u}} \cdot \delta \mathbf{u} \mathrm{d} \Omega\right]+\left[\int_{\Omega_{0} \backslash \Gamma_{0}} \mathbb{S}: \delta \mathbb{E} \mathrm{d} \Omega_{0}-\int_{\Gamma} \mathbf{T} \cdot \delta \boldsymbol{\delta} \mathrm{d} \Gamma\right]-\delta \Pi_{\mathrm{ext}}^{e}=0$

where $\mathbb{F}$ is the deformation gradient, $\mathbb{E}$ is the Green-Lagrange strain tensor, $\mathbb{S}$ is the second Piola-Kirchhoff stress tensor, $\rho$ is the material mass density, $\mathbf{u}$ are the displacements, $\ddot{\mathbf{u}}$ the accelerations, $\mathbf{T}=\sigma \mathbf{m}$ is the Cauchy traction vector on the interface ( $\sigma$ is the Cauchy stress tensor and $\mathbf{m}$ is the normal to the flank surface).

A solid-shell finite element discretization is adopted, by using the element proposed in [15] and successive modifications (in particular we use the selective mass scaling described in [3] and [4]). In the paper we assume the following numbering for the reference element: since the thickness is small, it is always possible to identify nodes 1-4 for the lower surface, and nodes 5-8 for the upper surface. By gathering the nodal coordinates in two vectors $\mathbf{X}_{1-4}$ and $\mathbf{X}_{5-8}$, it is possible to define as a corner fiber the segment connecting two corresponding nodes (one at the lower and one at the upper surface), henceforth coinciding with the element smaller edges. Fiber mid-points are therefore defined as:

$$
\mathbf{X}^{m}=\frac{\mathbf{X}_{1-4}+\mathbf{X}_{5-8}}{2}
$$

The DCE approach is based on the introduction, when a fracture criterion is satisfied at a solidshell finite element node, of a "cable" element either between two separating nodes (one of which is a duplicate of the original) or in correspondence of integration points along each one of the two crack flank middle surfaces (see figure ). The DCE, or "cable", element can interact with a blade, and transfer the contact forces to the opening faces in the correct direction, as shown in figure 1. 


\subsection{Cohesive force calculation}

The contribution to the internal energy of the cohesive forces acting on a separating element side can be expressed in terms of the nodal displacement jumps as

$$
\delta \Pi_{\text {coh,int }}^{e}=\int_{-1}^{+1} \int_{-1}^{+1} \mathbf{T} \cdot \delta\left[\sum_{a=1}^{4} N_{a}(\theta, \zeta) \boldsymbol{\delta}_{a}\right]^{e} J_{\Gamma} \mathrm{d} \theta \mathrm{d} \zeta
$$

where $\mathbf{T}$ is the traction vector obtained from the cohesive law, $J_{\Gamma}$ is the Jacobian determinant of the area transformation between the original and master reference system on the crack surface, $\theta$ and $\zeta$ are the intrinsic coordinates in the master reference system, along the crack direction and along the thickness direction, respectively. Therefore, $N_{a}(\theta, \zeta)$ represents the shape function of node $a$ restricted to the element face corresponding to the crack surface.

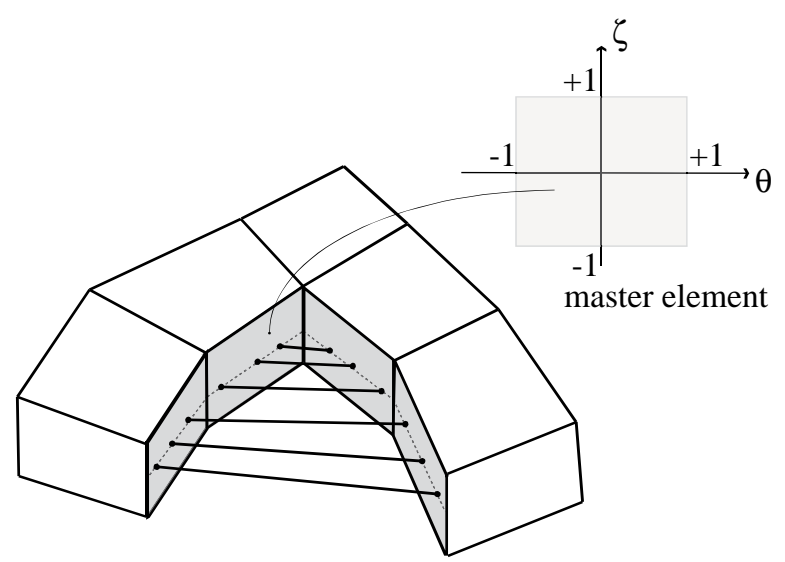

Figure 2: master element reference system for the DCEs inserted at the opening flank surfaces.

Cohesive forces are transmitted to the element side through a discrete number $N$ of of DCEs, introduced in correspondence of the adopted integration points. Only one integration point at $\zeta=0$ is assumed (i.e. in correspondence of the middle surface in the thickness direction) in view of the assumed small thickness, while along $\theta$ different choices are considered, as summarized in table 1. The implementation used in [6, 12] connects the DCEs to the fiber nodes at the crack flanks. This can be interpreted as adopting a NCI with a trapezoidal rule. In contrast, in this paper several alternatives for GI are explored. The contribution of the cracked element side to the cohesive internal work can be henceforth written as (without summation on repeated indices):

$$
\delta \Pi_{\text {coh, int }}^{e}=\sum_{a=1}^{4} \mathbf{f}_{a}^{e} \cdot \delta \boldsymbol{\delta}_{a}^{e}=\sum_{a=1}^{4} \sum_{k=1}^{N} w_{k} J_{\Gamma_{k}}^{e} \mathbf{T}_{k}^{e} N_{a}\left(\theta_{k}, \zeta=0\right) \cdot \delta \boldsymbol{\delta}_{a}^{e}
$$

where $\mathbf{f}_{a}^{e}=\sum_{k=1}^{N} w_{k} J_{\Gamma_{k}}^{e} \mathbf{T}_{k}^{e} N_{a_{k}}$ are the equivalent cohesive forces at each node of the opening face, $T_{k}$ is the cohesive traction transmitted by the cable at the $k$-th integration point, $w_{k}$ is the integration weight, $N_{a}\left(\theta_{k}, \zeta=0\right)$ is the shape function of node $a$ evaluated at the same position and $\delta \boldsymbol{\delta}_{a}$ is the virtual displacement jump at node $a$. 
Table 1: Gaussian or Newton-Cotes integration rules considered in the paper. $N$ is the number of integration points; $\theta_{k}, k=1, N$ are the master reference system element coordinates along the crack flank direction $(-1<\theta<+1)$; $w_{k}$ are the ordered weights used for the numerical integration.

\begin{tabular}{cccc}
\hline Integration rule & $N$ & $\theta_{k}$ & $w_{k}$ \\
\hline NCI & 2 & \pm 1 & 1,1 \\
GI1 & 1 & 0 & 2 \\
GI2 & 2 & \pm 0.577350269 & 1,1 \\
GI3 & 3 & $\pm 0.774596669,0$ & $5 / 9,8 / 9,5 / 9$ \\
GI4 & 4 & \pm 0.861136311594053, & $w_{a}, w_{b}, w_{b}, w_{a}$ \\
& & \pm 0.339981043584856 & $w_{a}=0.347854845137454$, \\
& & & $w_{b}=0.652145154862546$ \\
GI5 & 5 & \pm 0.906179845938664, & $w_{c}, w_{d}, w_{e}, w_{d}, w_{c}$ \\
& & $\pm 0.538469310105683,0$ & $w_{c}=0.236926885056189$, \\
& & & $w_{d}=0.478628670499366$, \\
& & & $w_{e}=0.56888888888889$ \\
\hline
\end{tabular}

The equivalent cohesive forces are then assembled together with the contributions of all the elements sharing the same node

$$
\mathbf{f}_{a}=\mathcal{A} \mathbf{f}_{a}^{e}=\mathcal{A} \sum_{k=1}^{N} w_{k} J_{\Gamma_{k}} \mathbf{T}_{k}^{e} N_{a}\left(\theta_{k}, \zeta=0\right)
$$

being $\mathcal{A}$ the assembly operator.

To guarantee force balance at the node at the time of node duplication, this force should be exactly equal to the force transmitted to node $a$ by the element on the other side of the crack, before node duplication and separation. At the insertion of the DCE, the nodes of the opening flanks are duplicated but they are still geometrically coincident. In the original implementation of the directional cohesive elements [12], the cohesive strength was initialized to match the component of the Cauchy stress normal to the crack flank at the integration point, consistent with the assumed mode I dominated opening. However, this procedure in general does not respect the force balance at the node. The sudden release of the unbalanced internal nodal forces upon node separation leads to oscillations in the stress field. A correction procedure in a single explicit dynamic time step has been envisaged as follows.

When the fracture activation criterion is met at a node, the opening face is identified as the one normal is closest to the direction of maximum principal stress. The node itself and the face are then duplicated and the topology of the elements belonging to the node support is updated. A prescribed number of directional cohesive elements is introduced between the two opening faces, depending on the chosen integration scheme. As already noticed in [9] and in [10], the duplication of a node causes a spurious stress oscillation due to the element-wise description of the crack propagation and to the release of internal nodal forces, which is only partially balanced by the cohesive force transmitted by the cable elements. In the framework of the XFEM method, to avoid this unphysical effect Menouillard and Belytschko [9, 10] proposed to introduce a fictitious nodal correction force exactly equal to the unbalanced part and linearly decaying in time.

The proposal in this work is to recompute the maximum tensile stress of the newly added directional cohesive elements, such that the equilibrium is restored at the time $t_{n d}$ of node duplication. Modifying this parameter has been shown [1, 17, 16] to not significantly alter the 
overall mechanical response, since the dissipative process is mainly governed by the fracture energy. At time $t_{n d}$, the initial cable lengths are zero and the directional cohesive elements are assumed to transmit a cohesive traction directed as the normal $\mathbf{m}_{k}$ to the opening element side at the $k$-th integration point and of magnitude $\mathbf{T}_{0_{k}}$, being $\mathbf{T}_{0_{k}}$ the maximum tensile stress at point $k$ (figure 3 ). Since at $t_{n d}$ the two duplicated nodes have the same velocity and acceleration, there are no jumps in the inertia and viscous forces. As a consequence, the equilibrium of node $a$ writes:

$$
\mathbf{f}_{a}=\mathcal{A} \sum_{k=1}^{N} w_{k} J_{\Gamma_{k}} \mathbf{T}_{0_{k}} N_{a_{k}}=\left(\mathbf{F}_{a}^{\mathrm{int}}-\mathbf{F}_{a}^{\mathrm{cohes}}\right) \cdot \mathbf{m}
$$

where $\mathbf{F}_{a}^{\text {int }}$ and $\mathbf{F}_{a}^{\text {cohes }}$ represent the internal nodal force and the cohesive force due to previously existent cables, computed after node duplication.

The cohesive forces are normal to the flank surfaces at the beginning; however, when the flanks start separating, then the cable tilts and shear forces are transmitted together with the normal ones. Morevover, the way the cohesive forces are transmitted to the crack flanks depends on whether the blade is interacting or not with the DCE. When the generic cable interacts with the blade through contact, the "directional" concept for the force calculation is triggered (see figure 1): if $\mathbf{x}_{k}^{c}$ is the position of the contact point, $\square^{+}$and $\square^{-}$indicate quantities at each (middle plane) flank surface, then the effective crack opening at $k$ is computed as

$$
\ell_{k}=\left\|\mathbf{x}_{k}^{c}-\mathbf{x}_{k}^{+}\right\|+\left\|\mathbf{x}_{k}^{c}-\mathbf{x}_{k}^{-}\right\|=\ell_{k}^{+}+\ell_{k}^{-} .
$$

The directions of the cohesive tractions transmitted by the cable element are aligned with the lines joining the contact points $\mathbf{x}_{C}$ to the positions of the integration points on the flank surface, namely:

$$
\mathbf{T}_{k}^{+}=T_{k} \mathbf{m}_{k}^{+} \quad \mathbf{T}_{k}^{-}=T_{k} \mathbf{m}_{k}^{-}
$$

where $T_{k}$ is the modulus of the cohesive traction computed through the cohesive law from the effective jump $\ell_{k}$ and

$$
\mathbf{m}_{k}^{+}=\frac{\mathbf{x}_{k}^{c}-\mathbf{x}_{k}^{+}}{\left\|\mathbf{x}_{k}^{c}-\mathbf{x}_{k}^{+}\right\|} \quad \mathbf{m}_{k}^{-}=\frac{\mathbf{x}_{k}^{c}-\mathbf{x}_{k}^{-}}{\left\|\mathbf{x}_{k}^{c}-\mathbf{x}_{k}^{-}\right\|}
$$

In these expressions, it is assumed that all the positions $\mathbf{x}_{k}$ are given, since they derive from the integration of the equations of motion.

In the absence of contact with a blade, the cohesive traction is simply:

$$
\mathbf{T}_{k}=\mathbf{T}_{k}^{+}=-\mathbf{T}_{k}^{-}=-\frac{\left\|\mathbf{T}_{k}\right\|}{\left\|\boldsymbol{\delta}_{k}\right\|} \boldsymbol{\delta}_{k} .
$$

A linear softening law, as depicted in figure 3, rules the magnitude of the cohesive force transmitted by the string element at the two opening faces as a function of the cable length. The area beneath the curve represents the fracture energy $\mathcal{G}_{c}=\frac{1}{2} T_{0} \ell_{c}$, being $\ell_{c}$ the critical length corresponding to the complete decohesion of the two flanks and $T_{0}$ being determined from (6).

\section{NUMERICAL EXAMPLES}

\subsection{Three point bending specimen}

Let us consider a three point bending specimen [5, 2], whose dimensions are shown in figure

4. Several discretizations based on solid-shell elements, with out-of-plane thickness $B=5$ 


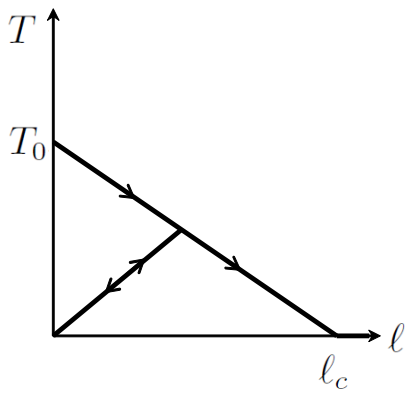

Figure 3: linear softening law adopted for the DCE.

$\mathrm{mm}$, are explored, each one identified by a vertical dimension of the finite element in the set $h_{e}=\{7.5,10,15,25,30\} \mathrm{mm}$ constant along the beam height (in figure 4 the case $h_{e}=30 \mathrm{~mm}$ is depicted); the finite element width is instead always fixed to $10 \mathrm{~mm}$. The material properties are taken from [5] and are the following: Young's modulus $E=36,500 \mathrm{MPa}$, Poisson's ratio $\nu=0.1$, cohesive strength $T_{0}=3.19 \mathrm{MPa}$ and mode I fracture energy $\mathcal{G}_{c}=0.05 \mathrm{~N} / \mathrm{mm}$. With these parameters the characteristic length of the cohesive process zone is $\ell_{p z}=E \mathcal{G}_{c} / T_{0}^{2}=179$ $\mathrm{mm}$, therefore larger than the beam height. Consequently, every case discussed in this section largely satisfies the condition on the minimum number of integration points in the cohesive process zone, e.g. a minimum of 3-5 linear finite elements. The analyses are carried out using an explicit dynamics approach in displacement control, with the top middle point of the beam moving downwards with a constant velocity of $2 \mathrm{~mm} / \mathrm{s}$. A Rayleigh damping has been added to limit the oscillations due to inertial effects in the bulk material and to mimic a quasi-static response.

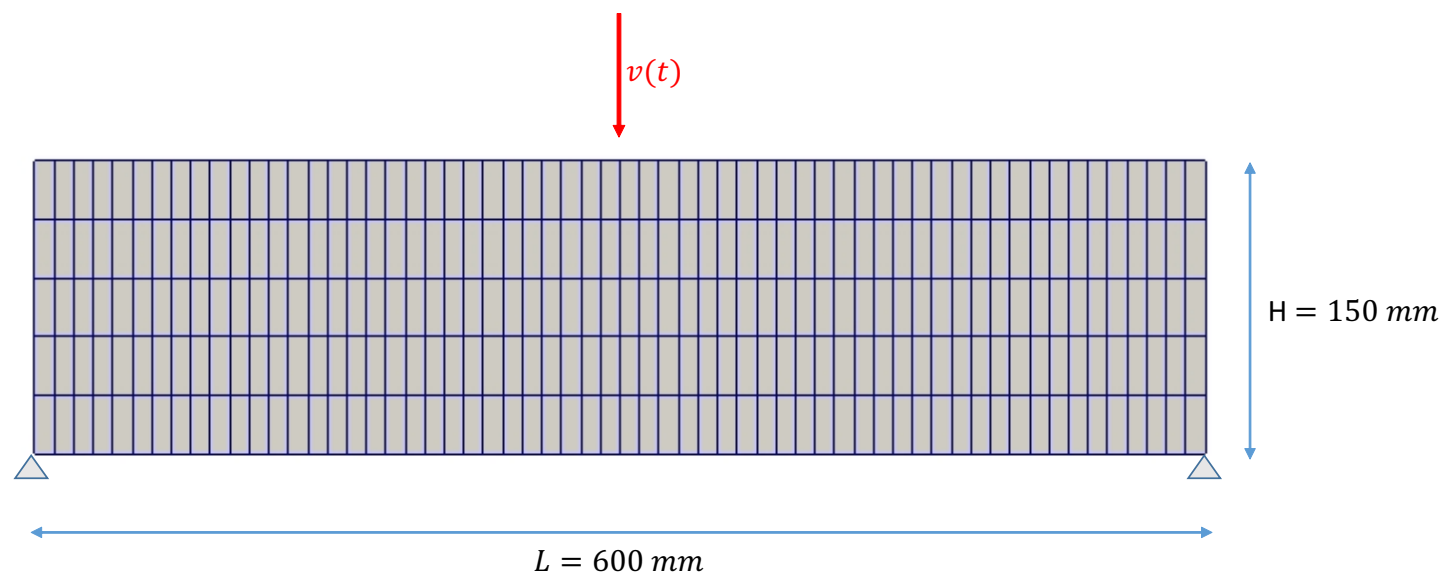

Figure 4: three point bending specimen, (coarser) mesh size $h_{e}=30 \mathrm{~mm}$.

To evaluate the influence of the mesh size, the results are shown in figure 5 in terms of dimensionless load-displacement curves and accelerations vs the dimensionless vertical, imposed 
displacement. In these examples, only one cable is inserted between the opening faces. It can be seen that in all the cases a sudden decrease of the load corresponds to the snap-back experimental behaviour. The mesh sizes $h_{e}=25,30 \mathrm{~mm}$ show a pathological behaviour in the post-peak regime, partially explained by the damping factor adopted. The accelerations reported in the graph at the bottom in figure 5, are strongly influenced by the discretizations in space and time. However, the steep peaks registered have different interpretation: before the maximum load value they indicate the opening of the first crack faces and cable insertion during loading; then there are acceleration peaks, despite damping, in correspondence of the large load drop because of the structural snap-back, whose disturbance decreases in a not negligible time interval; in the final, descending part of the curves, the accelerations of the node at the crack mouth are related, instead, to the opening of the elements along the ligament, before the crack reaches the last element at the specimen top (in the model, this element, where the displacement is imposed, cannot be broken). When a DCE reaches its maximum elongation $\ell_{c}$ and disappears, there are no appreciable disturbances, since the exchanged cohesive forces in that case are already almost zero.
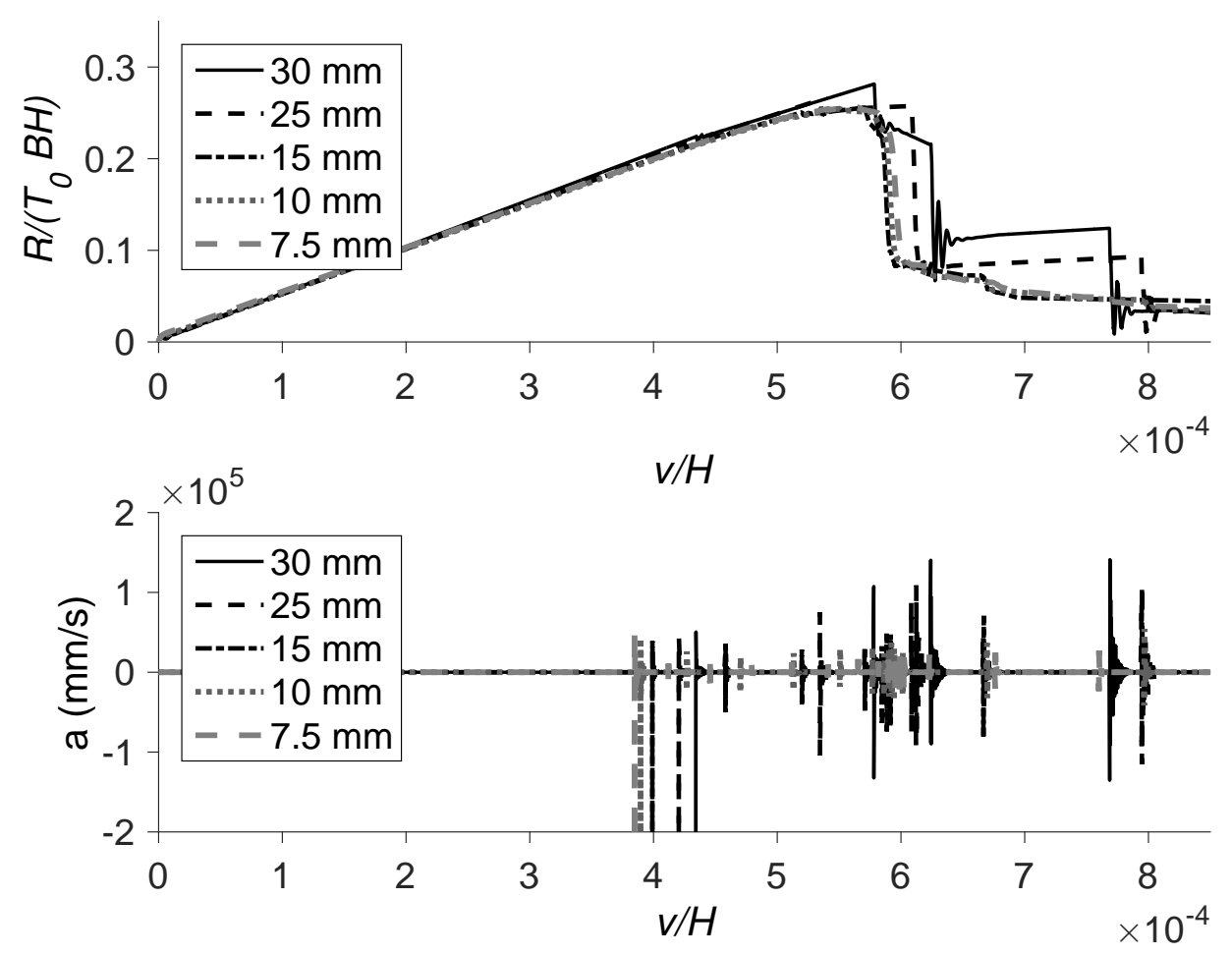

Figure 5: three point bending specimen. Comparison in terms of load displacement response and acceleration at the crack mouth between different meshes (one cable per element case).

To evaluate the influence of the number of the DCEs inserted for each solid-shell element opening face, we present in figure 6 the cases $h_{e}=15,30 \mathrm{~mm}$. Since the test is carried out with displacement control, the sudden load drop can be well reproduced even with the coarse discretization if two cables per opening face are inserted. While not reported here, the addition of more cables does not improve the response, since the element size is sufficiently smaller than the cohesive length as in this case. Since the opening face can change its configuration 
according to the displacements at its nodes and the cables are inserted at the face middle plane, the crack opening profile is linear and the transmitted cohesive traction is already accurately described by two cables.

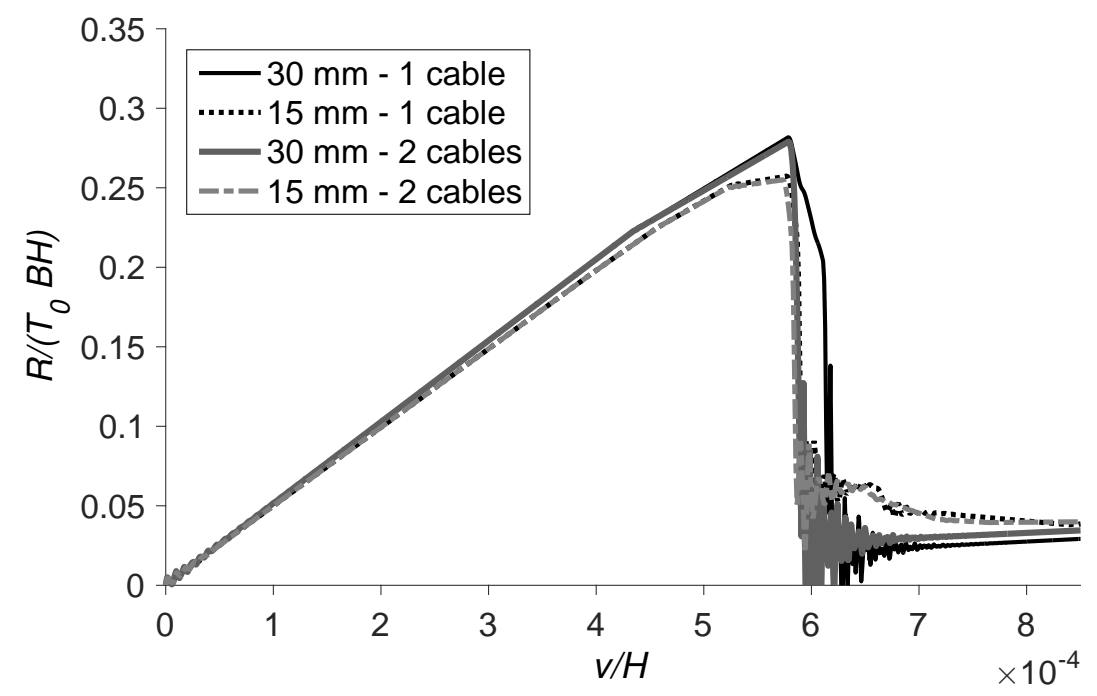

Figure 6: Three point bending specimen. Comparison of load displacement curves for different number of cables, $h_{e}=15 \mathrm{~mm}$.

All the previous results have been obtained using the correction approach defined in (6). To point out its role, in figure 7 we compare the outcomes with and without correction for the case $h_{e}=15 \mathrm{~mm}$. It can be noticed that the introduction of the correction procedure allows to obtain a smooth response, avoiding the unphysical jumps that can be observed in the curve without correction. The entity of the correction is slightly different for each DCE along the crack propagation direction: the maximum correction value for $T_{0}$ is an increasing of about $40 \%$ for the one cable case; the entity of the correction, however, is smaller when the number of the cables is increased.

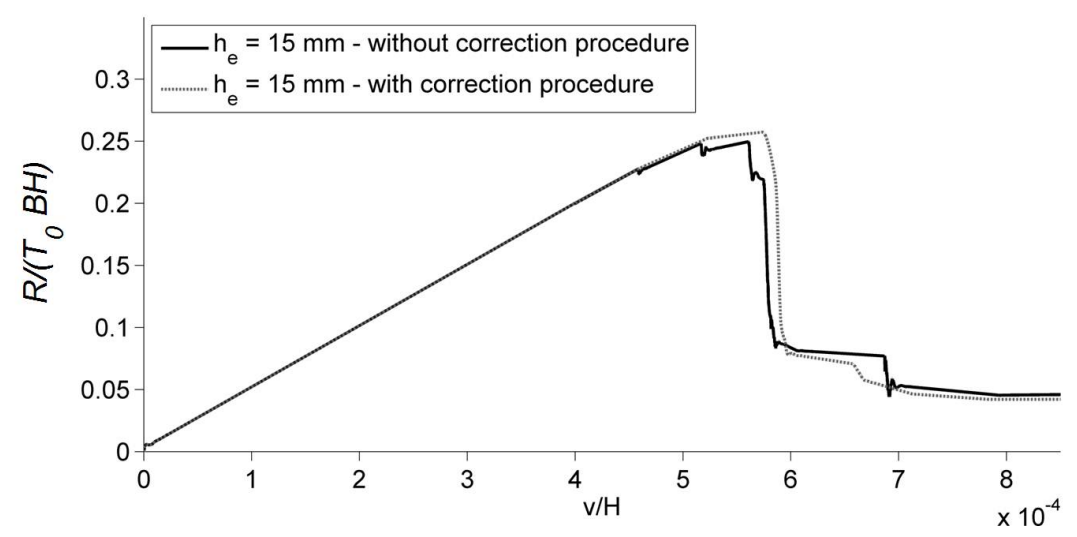

Figure 7: Three point bending specimen. Comparison of load displacement curves for different number of cables, $h_{e}=15 \mathrm{~mm}$. 


\subsection{Coarse regular mesh with a rectilinear crack}

In this section we adapt the example from [10], where brittle fracture was considered (no cohesion), to the present DCE approach with solid-shell elements in explicit dynamics, with the proposed correction of the cohesive strength, according to equation (6). A rectilinear crack is propagated in a coarse and regular mesh with an imposed advancing crack tip velocity of 1 $\mathrm{m} / \mathrm{s}$ (figure $8 \mathrm{a}$ ). The bottom nodes of the model are restrained, while at the top edge a constant stress $\sigma=10 \mathrm{kPa}$ is imposed. Dimensions are the following: length of the specimen $L=0.2$ m, width $H=0.1 \mathrm{~m}$, initial crack length $a_{i}=0.04 \mathrm{~m}$. The material properties for the bulk are linear elastic, with Young's modulus $E=1 \mathrm{MPa}$, Poisson's ratio $\nu=0.3$, and mass density $\rho=1 \mathrm{~kg} / \mathrm{m}^{3}$. The cohesive strength is $T_{0}=10 \mathrm{kPa}$, and $\mathcal{G}_{c}=0.05 \mathrm{~N} / \mathrm{mm}$. The damping parameter is set to $2 \%$.

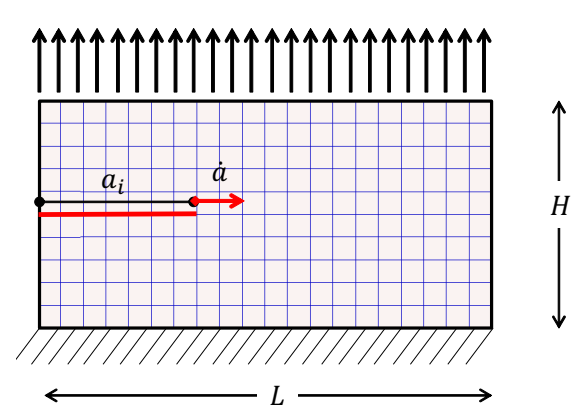

(a)

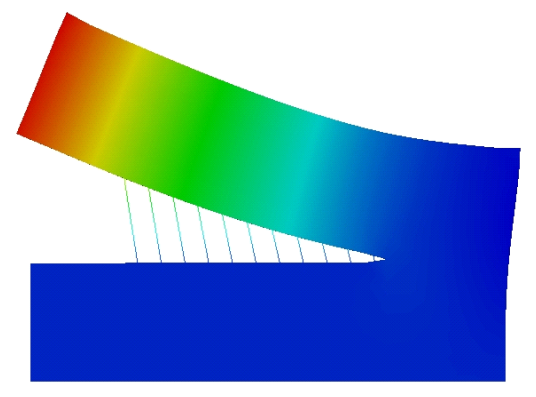

(b)

Figure 8: rectilinear crack propagation. (a) geometry, load and boundary conditions and (b) deformed shape.

Spurious oscillations at the top left corner node, induced by the sudden insertion of the cohesive elements at the nodes of the element near the crack tip, are monitored. In figure $9 \mathrm{a}$ the displacement vs time response appears smoothed when the correction is activated; it is instead strongly discontinuous without correction. The effect of the correction becomes more evident as the time increases, i.e. when the crack is far from the top left vertex node during the propagation. It should be emphasized the the different response is obtained using in the two analyses cohesive models with the same fracture energy. The only difference is the initial value of the traction $T_{0}$ that in the case without correction is taken equal to the normal component of Cauchy stress at the time $t_{n d}$ of node duplication, while in the case of the correction is determined according to (6). In figure 9b the velocity at the same point is depicted: the correction decreases the peaks also in this case. It is worthwhile to emphasize that, without correction, the induced velocities appear completely not physical; when we include the correction, the values turn out to be much smaller and acceptable.

\section{CONCLUSIONS}

In this paper some progresses on the development of the directional cohesive finite element approach have been presented, including: i) the possibility to increase the number of inserted cables for each opening face of solid-shell finite elements, and ii) corrected definition of maximum cohesive tractions based on nodal equilibrium to avoid the spurious oscillations in the local stress field arising from the insertion of cohesive finite elements at the crack tip. These numerical improvements can be helpful during the simulation of sharp blades cutting through 


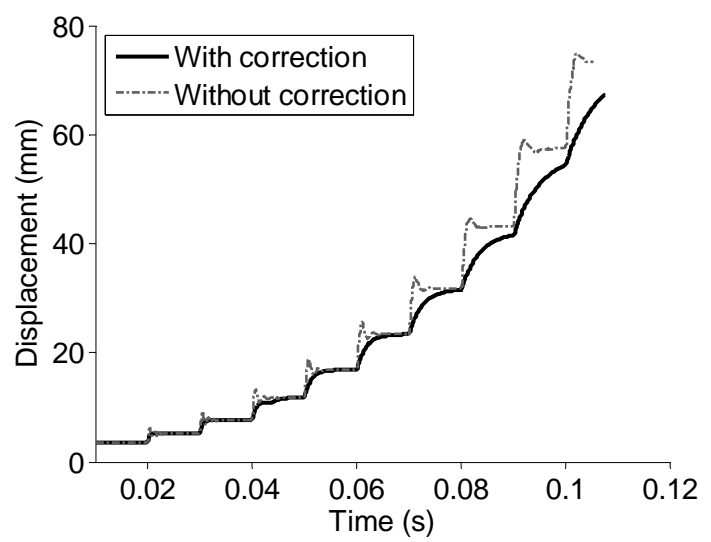

(a)

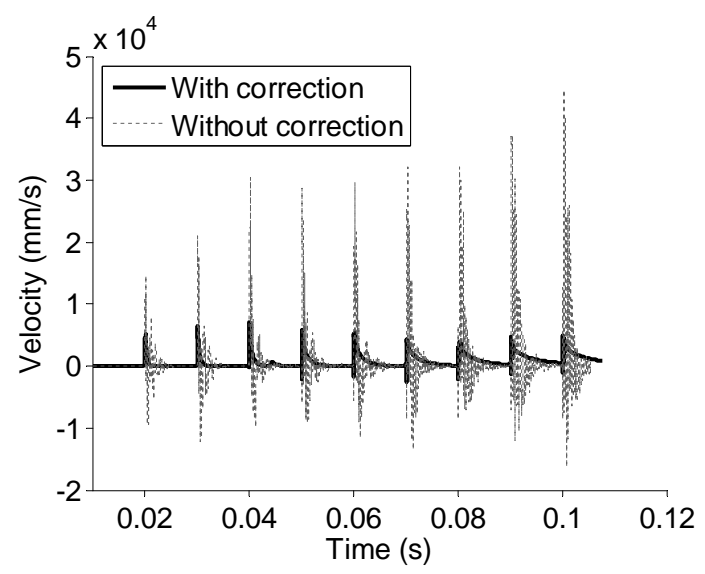

(b)

Figure 9: rectilinear crack propagation. Comparisons for corrected and not corrected responses in terms of (a) vertical displacement and (b) velocity at the top left corner vertex.

layered shells, where the cohesive zone length is of the order of the curvature radius of the cutting blade. We showed, through numerical examples taken from the literature, how different choices influence the numerical results; in particular, the beneficial role of corrected nodal cohesive forces to smooth numerical oscillations at the insertion of cohesive elements in explicit dynamics problems is emphasized.

\section{ACKNOWLEDGEMENTS}

The financial support by Tetra Pak Packaging Solutions is kindly acknowledged.

\section{REFERENCES}

[1] G. Alfano and M. A. Crisfield. Finite element interface models for the delamination analysis of laminate composites: mechancial and computational issues. International Journal for Numerical Methods in Engineering, 50:1701-1736, 2001.

[2] A. Carpinteri and G. Colombo. Numerical analysis of catastrophic softening behaviour (snap-back instability). Computers \& Structures, 31:607 - 636, 1989. 
[3] G. Cocchetti, M. Pagani, and U. Perego. Selective mass scaling for distorted solid-shell elements in explicit dynamics: optimal scaling factor and stable time step estimate. International Journal for Numerical Methods in Engineering, 101(9):700-731, 2015. nme.4829.

[4] F. Confalonieri, A. Ghisi, and U. Perego. 8-node solid-shell elements selective mass scaling for explicit dynamic analysis of layered thin-walled structures. Computational Mechanics, 56(4):585-599, 2015.

[5] B.C. Do, W. Liu, Q.D. Yang, and X.Y. Su. Improved cohesive stress integration schemes for cohesive zone elements. Engineering Fracture Mechanics, 107:14 - 28, 2013.

[6] A. Frangi, M. Pagani, U. Perego, and R. Borsari. Directional Cohesive Elements for the Simulation of Blade Cutting of Thin Shells. Computer Modeling in Engineering \& Sciences, 57:205-224, 2010.

[7] A Giampieri and U Perego. An interface finite element for the simulation of localized membrane-bending deformation in shells. Computer Methods in Applied Mechanics and Engineering, 200:2378-2396, 2011.

[8] P. Gilormini and J. Diani. Testing some implementations of a cohesive-zone model at finite strain. Engineering Fracture Mechanics, 148:97 - 109, 2015.

[9] T. Menouillard and T. Belytschko. Correction force for releasing crack tip element with xfem and only discontinuous enrichment. European Journal of Computational Mechanics, 18(5-6):465-483, 2009.

[10] T. Menouillard and T. Belytschko. Smoothed nodal forces for improved dynamic crack propagation modeling in XFEM. International Journal for Numerical Methods in Engineering, 84:47-72, 2010.

[11] M. Ortiz and A. Pandolfi. Finite-defomation irreversible cohesive elements for threedimensional crack propagtion analysis. International Journal for Numerical Methods in Engineering, 44:1267-1282, 1999.

[12] M. Pagani and U. Perego. Explicit dynamics simulation of blade cutting of thin elastoplastic shells using a "directional" cohesive elements in solid-shell finite element models. Computer Methods in Applied Mechanics and Engineering, 285:515 - 541, 2015.

[13] H. Qiao, W. Q. Chen, Q.D. Yang, and J. Lua. Augmented Cohesive Elements for Efficient Delamination Analyses of Composite Laminates. Journal of Engineering Materials and Technology, 133:041010-1, 2011.

[14] J. C. J. Schellekens and R. De Borst. On the numerical integration of interface elements. International Journal for Numerical Methods in Engineering, 36:43-66, 1993.

[15] M. Schwarze and S. Reese. A reduced integration solid-shell finite element based on the eas and the ans concept: large deformation problems. International Journal for Numerical Methods in Engineering, 85(3):289-329, 2011.

[16] A. Turon, P. P. Camanho, J. Costa, and J. Renart. Accurate simulation of delamination growth under mixed-mode loading using cohesive elements: Definition of interlaminar strengths and elastic stiffness. Composite Structures, 92(8):1857-1864, 2010. 
[17] A. Turon, C. G. Dávila, P. P. Camanho, and J. Costa. An engineering solution for mesh size effects in the simulation of delamination using cohesive zone models. Engineering Fracture Mechanics, 74(10):1665-1682, 2007.

[18] Q. D. Yang, X. J. Fang, J. X. Shi, and J. Lua. An improved cohesive element for shell delamination analyses. International Journal for Numerical Methods in Engineering, 83(5):611-641, 2010. 